\title{
Decline of Renal Function and Progression of Left Ventricular Hypertrophy Are Independently Determined in Chronic Kidney Disease Stages 3-5
}

\author{
Hiromichi Suzuki ${ }^{a}$ Tsutomu Inoue ${ }^{a}$ Mami Dogic Tomohiro Kikuta ${ }^{\text {a }}$ \\ Tsuneo Takenakab Hirokazu Okada ${ }^{a}$ \\ a Department of Nephrology, Saitama Medical University, and bepartment of Nephrology, \\ International University of Health and Welfare, Medical University Hospital, Saitama, and \\ ${ }^{c}$ Omron Health Care Co. Ltd., Kyoto, Japan
}

\section{Key Words}

Central aortic pressure - Estimated glomerular filtration rate $\cdot$ Blood pressure $\cdot$ Pulse wave velocity

\begin{abstract}
Invasive and noninvasive methods for evaluating the effects of hemodynamics on progression of left ventricular hypertrophy (LVH) in patients with chronic kidney disease (CKD) have been proposed. Central aortic pressure (CAP) has been reported to be the best among selected measures of hemodynamics for predicting LVH. However, there are few studies examining the relation between longitudinal changes in CAP and renal dysfunction in patients with CKD. Methods: Sixty-seven patients with CKD stages 3-5 (female/male ratio: 26/41, age: $61.5 \pm 13.1$ years) were followed for 5 years. Before and at the end of the 5 -year period, CAP was recorded by an automated tonometric system (HEM-9000 AI; Omron Healthcare, Kyoto, Japan). Second systolic aortic blood pressure (SBP2) was employed as an index of CAP. Results: Throughout the study, systolic blood pressure (SBP) was well controlled. Renal function followed by estimated glomerular filtration rate (eGFR) gradually worsened as a whole. Ten patients had renal replacement therapy, 3 patients developed cardiovascular diseases and 2 patients were found to have a neoplasm during the 5 -year observation period. SBP2 increased from $120 \pm$ 19 to $125 \pm 33 \mathrm{~mm} \mathrm{Hg}$ and eGFR decreased from $38.2 \pm 18.2$ to $29.5 \pm 16.3 \mathrm{ml} / \mathrm{min} / 1.73 \mathrm{~m}^{2}$; however, these differences did not achieve significance. The left ventricular mass (LVM) index significantly increased from $115.5 \pm 10.5$ to $131.2 \pm 11.7 \mathrm{~g} / \mathrm{m}^{2}$ ( $\left.p<0.05\right)$. Although the changes in SBP2 and eGFR looked like a mirror image, there was no significant correlation between the two factors. Moreover, multivariate regression analysis did not reveal a close correlation between SBP2 and CKD progression. In contrast to the decline of renal function, the baseline
\end{abstract}


Suzuki et al.: Decline of Renal Function and Progression of Left Ventricular

Hypertrophy Are Independently Determined in Chronic Kidney Disease Stages 3-5

value of SBP2 predicted an increase in the LVM index. Conclusion: Worsening of renal dysfunction is not solely dependent on hemodynamics. Other factors might be involved in a complex manner.

(c) 2014 S. Karger AG, Basel

\section{Introduction}

In patients with chronic kidney disease (CKD), hypertension is closely associated with left ventricular hypertrophy (LVH). LVH is caused by a chronically increased workload on the heart [1] by hypertension and is a strong predictor of mortality in patients with CKD [2]. According to data from the Chronic Kidney Disease Japan Cohort (CKD-JAC) study [3], independent risk factors for LVH were a history of cardiovascular disease (CVD), systolic blood pressure (SBP), body mass index, and serum calcium level. Since nearly half of the patients had diabetes mellitus (DM), the findings of this study might be different than those for non-DM patients. In non-DM patients with a progressive decline of renal function, it has been proposed that pulse pressure (PP), which is known as a marker of stiffness of large arteries [4], is the sole predictor of LVH [5]. In patients with essential hypertension, Verdecchia et al. [6] demonstrated that SBP mainly contributed to the association between PP and LVH. Compared with hypertensive patients, biochemical factors are known to be additionally involved in the progression of LVH in patients with CKD [2].

In the vasculature of patients with CKD, arterial stiffness is a composite product of pressure-mediated damage and biochemical alterations [7]. Several studies provided evidence that aortic stiffness plays a role in the decline of renal function [8, 9]. Moreover, increased local arterial stiffness increases the pressure ratio of the forward wave which affects the central aortic pressure (CAP). Recently, Ng et al. [10] have reported the relationship between CAP and the left ventricular mass (LVM) index and pulse wave velocity (PWV) in non-DM patients with CKD and in renal transplant recipients. This cross-sectional study demonstrated that a change in CAP could only be explained by peripheral PP but not by the LVM index and PWV.

The aim of the present longitudinal study was to evaluate the relation between the changes in CAP and LVM index and renal dysfunction in patients with CKD stages 3-5.

\section{Patients and Methods}

This was a prospective, observational, single-center cohort study. This study was conducted in accordance with the Declaration of Helsinki. Approval for the study was obtained from the Saitama Medical University Ethics Committee, and written informed consent was obtained from each participant.

Patients with CKD stages 3-5 [estimated glomerular filtration rate (eGFR) $60-0 \mathrm{ml} / \mathrm{min} / 1.73 \mathrm{~m}^{2}$ without dialysis] [11] were recruited from specialist renal clinics at the Renal Disease Center, Saitama Medical University from October 2006 to March 2007. All participating patients were followed for 5 years or until death occurred, dialysis therapy was started, renal transplantation was performed, neoplasm was found, or cardiovascular events occurred (fatal or nonfatal myocardial infarction, cerebrovascular diseases, or aortic dissection). Inclusion criteria were CKD stages 3-5 as defined by K/DOQI [12], not yet being on dialysis, stable renal function within the last 3 months, $<5 \mathrm{ml} / \mathrm{min} / 1.73 \mathrm{~m}^{2}$ change in eGFR, and no change in medication in the preceding 3 months.

Exclusion Criteria

Exclusion criteria included DM, atrial fibrillation, known left ventricular dysfunction (ejection fraction $<55 \%$ ), or signs and symptoms of congestive heart failure, pregnancy or lactation, significant valvular or coronary heart disease, cardiac arrhythmia or conduction defects, systemic diseases, proteinuria in the nephrotic range $(<3.0 \mathrm{~g} /$ day), and use of sedative or hypnotic drugs or any other drugs potentially affecting blood pressure (BP) during ambulatory monitoring, e.g. corticosteroids. 
The serum creatinine, urinary excretion of creatinine and protein, and hematologic and serum tests including urea, uric acid, blood urea nitrogen, electrolytes, etc., were obtained at the beginning of the observation period, during every month of follow-up, and at the end of the observation period. eGFR was calculated using a modified 3-variable equation for eGFR in Japanese patients: eGFR $=194 \times \mathrm{age}^{-0.287} \times \mathrm{sCr}^{-1.094}(\times 0.739$, if female), where $\mathrm{sCr}=$ serum creatinine [13].

\section{Cause of Renal Impairment}

The cause of CKD was assessed by a review of the clinical history and other investigations. Patients were classified as 'hypertensive/glomerulosclerosis' if they had no clear evidence of active renal disease but had a history of hypertensive diseases and a positive urinary protein excretion without casts. The underlying etiologies of CKD in our study patients included chronic glomerulonephritis in 51 patients (76\%), hypertensive nephrosclerosis in 13 patients (19\%), and other diseases in 3 patients. All patients were treated with antihypertensive drugs including renin-angiotensin inhibitors, and their blood pressure was well controlled.

\section{BP Measurements}

$\mathrm{BP}$ was not measured in the clinic; patients were given instructions on how to measure and record their own BP at home. BP measurements were recorded at least twice a week at home in the sitting position - once in the morning before breakfast within $30 \mathrm{~min}$ of awakening and once in the evening just before dinner. Home BP measurements were made using the HEM 401C (Omron Life Science Co. Ltd., Tokyo, Japan), a semiautomatic device that operates on the cuff-oscillometric principle and generates a digital display of SBP and diastolic blood pressure (DBP) and pulse rate [14].

\section{Measures of PWV and Second Systolic Aortic Blood Pressure}

Second systolic aortic blood pressure (SBP2) was defined as an index of CAP. PWV was measured using an automatic waveform analyzer (form PWV/ABI; Omron Colin, Co., Ltd., Komaki, Japan). All individuals were examined after resting in the supine position for at least $5 \mathrm{~min}$, and radial artery pulse waveform was recorded by an automated tonometric system (HEM-9000 AI; Omron Healthcare, Kyoto, Japan) with patients in a sitting position. SBP2 was well correlated with CAP, which was measured simultaneously by the direct catheter method [13-15]. Two measurements were taken 5 min apart, and their average was used in the analysis.

\section{Echocardiography}

Left ventricular end-diastolic and end-systolic diameter, ventricular septal wall thickness, and left posterior wall thickness were assessed by M-mode echocardiography after selecting the measurement section by B-mode echocardiography. The LVM index was calculated according to the Penn formula. Data were averaged over 5 cardiac cycles $[15,16]$. Echocardiography was performed before the start of the study and at the end of the 5-year study. The interobserver agreement was $90 \%$ and the intraobserver agreement was $91 \%$.

\section{Statistical Analysis}

Statistical analyses were performed using JMP software, version 9 (JMP, SAS, Cary, N.C., USA). Correlations between various characteristics were determined using Pearson's correlation test. $\mathrm{p}<0.05$ was considered significant. Univariate analysis for the decline of renal function as evaluated by eGFR and for the increase in LVM was used to assess the relationship between the differences in eGFR or the increase in LVM and selected variables. Parameters reaching a significance level of $p<0.05$ were entered into both forward and backward selection algorithms. Multiple linear regression analysis was used to examine the independent predictors of differences in eGFR or the increase in LVM index.

Patient event-free curves were calculated by the Kaplan-Meier life-table analysis method, and differences between the groups were evaluated by the log-rank test. Values are given as means \pm SD. The significance of differences in the echocardiographic and tonometric data at baseline and after 5 years was assessed by paired $t$ tests. $p$ values $<0.05$ were regarded as statistically significant.

\section{Results}

Sixty-seven patients with CKD stages 3-5 were enrolled in this study. Their baseline characteristics are summarized in table 1. 


\begin{tabular}{|c|c|}
\hline \multicolumn{2}{|l|}{ Pulse 2014;2:29-37 } \\
\hline DOI: $10.1159 / 000368678$ & $\begin{array}{l}\text { (c) } 2014 \text { S. Karger AG, Basel } \\
\text { www.karger.com/pls }\end{array}$ \\
\hline \multicolumn{2}{|c|}{$\begin{array}{l}\text { Suzuki et al.: Decline of Renal Function and Progression of Left Ventricular } \\
\text { Hypertrophy Are Independently Determined in Chronic Kidney Disease Stages 3-5 }\end{array}$} \\
\hline Age, years & $61.5 \pm 13.1$ \\
\hline Gender, male/female & $41 / 26$ \\
\hline eGFR, $\mathrm{ml} / \mathrm{min} / 1.73 \mathrm{~m}^{2}$ & $31.3 \pm 16.7$ \\
\hline SBP, mm Hg & $136.1 \pm 21.4$ \\
\hline DBP, mm Hg & $77.0 \pm 14.1$ \\
\hline $\mathrm{PP}, \mathrm{mm} \mathrm{Hg}$ & $62.1 \pm 8.2$ \\
\hline $\mathrm{HR}$, beats/min & $74.1 \pm 10.9$ \\
\hline baPWV, cm/s & $1,560.7 \pm 325.7$ \\
\hline SBP2, mm Hg & $128.3 \pm 19.5$ \\
\hline LVM index & $116.4 \pm 10.7$ \\
\hline Total cholesterol, mg/dl & $201.2 \pm 36.3$ \\
\hline Phosphate, $\mathrm{mg} / \mathrm{dl}$ & $4.6 \pm 0.7$ \\
\hline Calcium, mg/dl & $9.0 \pm 1.0$ \\
\hline Urinary protein excretion, $\mathrm{g} / \mathrm{gCr}$ & $1.23 \pm 0.92$ \\
\hline Serum albumin, g/dl & $4.0 \pm 0.4$ \\
\hline Hemoglobin, g/dl & $11.2 \pm 0.9$ \\
\hline
\end{tabular}

Table 1. Baseline characteristics and laboratory findings of the patients with CKD stages 3-5 ( $\mathrm{n}=67)$

\author{
Age, years \\ Gender, male/female \\ eGFR, $\mathrm{ml} / \mathrm{min} / 1.73 \mathrm{~m}^{2}$ \\ SBP, $\mathrm{mm} \mathrm{Hg}$ \\ PP, $\mathrm{mm} \mathrm{Hg}$ \\ Reats $/ \mathrm{min}$ \\ SBP2, $\mathrm{mm} \mathrm{Hg}$ \\ LVM index \\ Phosphate, mg/dl \\ Calcium, mg/dl \\ Serum albumin, $\mathrm{g} / \mathrm{d}$
}

$4.0 \pm 0.4$

$.5 \pm 13.1$

$31.3 \pm 16.7$

$36.1+21.4$

$01.2 \pm 36.3$

Table 2. Comparison of baseline parameters among patients who developed ESRD, patients who suffered events (CVD or neoplasm), and the other patients who were followed for the entire 5-year period $(n=52)$

\begin{tabular}{lccc}
\hline Variables & $\begin{array}{c}\text { Patients followed } \\
\text { for 5 years (n= 52) }\end{array}$ & $\begin{array}{l}\text { Patients who developed } \\
\text { ESRD }(\mathrm{n}=10)\end{array}$ & $\begin{array}{c}\text { Patients who suffered } \\
\text { events }(\mathrm{n}=5)\end{array}$ \\
\hline Age, years & $61.5 \pm 13.1$ & $61.5 \pm 13.7$ & $62.3 \pm 14.2$ \\
eGFR, ml/min/1.73 m² & $40.2 \pm 15.7$ & $25.7 \pm 15.1^{*}$ & $22.72 \pm 16.0$ \\
SBP, mm Hg & $131.9 \pm 15.7$ & $141.2 \pm 25.6$ & $146.3 \pm 28.1$ \\
DBP, mm Hg & $75.7 \pm 13.0$ & $78.06 \pm 14.95$ & $74.37 \pm 17.59$ \\
PP, mm Hg & $56.4 \pm 14.6$ & $66.9 \pm 24.8$ & $66.5 \pm 18.4$ \\
HR, beats/min & $73.1 \pm 11$ & $76.6 \pm 9.1$ & $74.5 \pm 8.7$ \\
baPWV, cm/s & $1,545.9 \pm 316$ & $1,637.5 \pm 349$ & $2,108.0 \pm 394$ \\
SBP2, mm Hg & $126.2 \pm 20.2$ & $132.5 \pm 29.5$ & $141.1 \pm 36.0$ \\
LVM index, g/m 2 & $114.2 \pm 10.9$ & $112.3 \pm 11.8$ & $128.6 \pm 19.5$ \\
Albumin, g/dl & $4.1 \pm 0.5$ & $4.0 \pm 0.4$ & $4.0 \pm 0.6$ \\
Hemoglobin, g/dl & $11.8 \pm 1.6$ & $11.0 \pm 0.9$ & $10.5 \pm 1.2$ \\
Total cholesterol, mg/dl & $185.9 \pm 34.8$ & $200.5 \pm 36.3$ & $216.2 \pm 29.7$ \\
Calcium, mg/dl & $9.0 \pm 0.41$ & $8.9 \pm 1.0$ & $9.1 \pm 1.2$ \\
Phosphate, mg/dl & $4.56 \pm 0.73$ & $4.89 \pm 0.7$ & $4.1 \pm 0.9$ \\
Urinary protein excretion, g/g Cr & $1.09 \pm 0.92$ & $1.56 \pm 1.22$ & $0.54 \pm 0.92$ \\
\hline
\end{tabular}

$\mathrm{n}=$ Number of patients followed for 5 years; $\mathrm{Cr}=$ creatinine.

* Indicates $\mathrm{p}<0.05$ compared with the patients followed for 5 years.

Fifty-two patients did not develop CVD or neoplasm, did not receive dialysis, and did not undergo renal transplantation during the 5-year follow-up period. A comparison of parameters measured at baseline among patients who developed end-stage renal disease (ESRD), patients who developed CVD or neoplasm, and the other 52 patients who were followed for the entire 5-year period is shown in table 2. The baseline value of eGFR was significantly lower in patients who progressed to ESRD, and there were no significant differences in other parameters among the 3 groups.

The changes in SBP, DBP, PP, eGFR, brachial-ankle PWV (baPWV), heart rate (HR), SBP2, and LVM index of the 52 patients at the start and at the end of the 5-year observation period are shown in figures $1-3$. Except for the LVM index $(\mathrm{p}<0.05)$, there were no significant differences in the parameters at the two time points. Kaplan-Meier curves of the patients are 


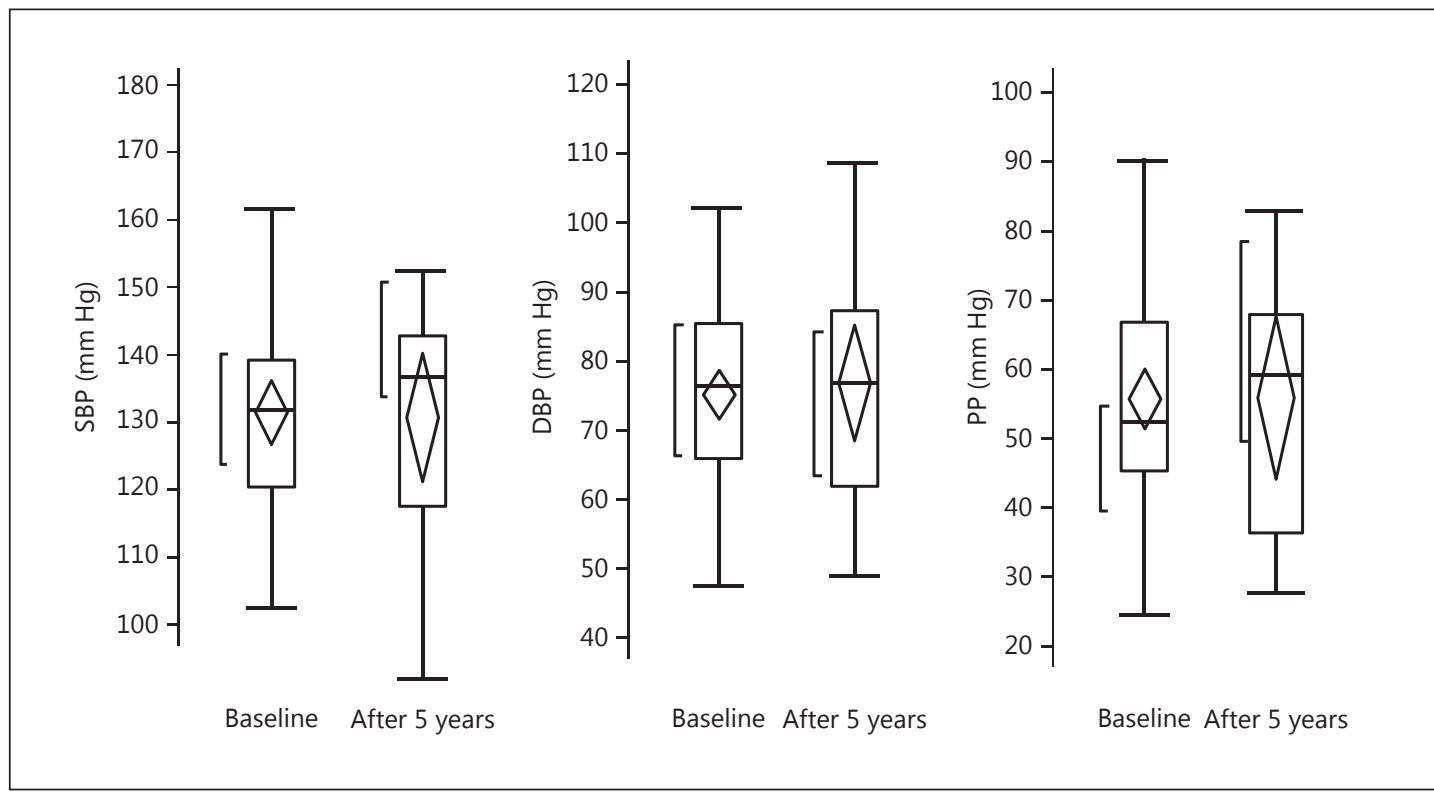

Fig. 1. Changes in SBP, DBP and PP. There were no significant changes in SBP, DBP and PP between patients at the start and those at the end of the 5-year observation period $(n=52)$.

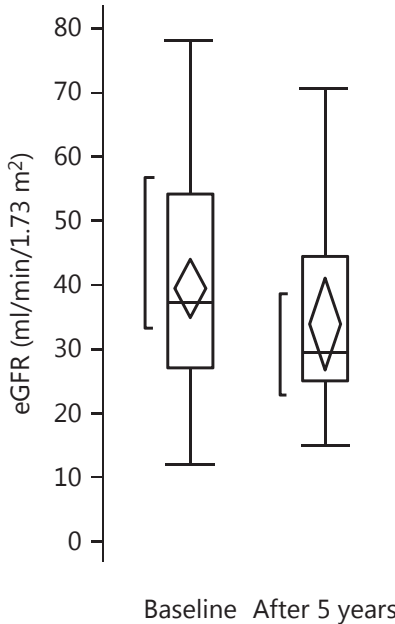

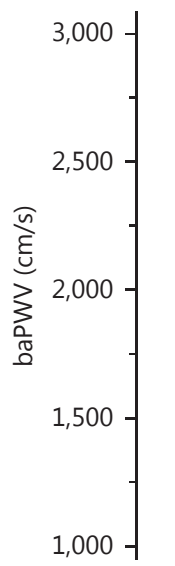

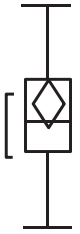

Baseline After 5 years

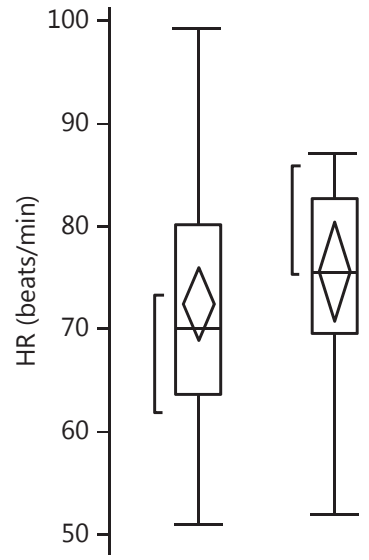

Baseline After 5 years

Fig. 2. Changes in eGFR, baPWV and HR. There were no significant changes in eGFR, baPWV and HR between patients at the start and those at the end of the 5 -year observation period $(n=52)$.

shown in figure 4 . The incidence-free rates at 1 year and 5 years were 90 and 78\%, respectively.

Tables 3 and 4 display the results of the univariate analysis of demographic variables on decline in renal function and increase in LVM index, respectively, in the 52 patients who were followed for the 5-year period. In the univariate analysis, the increased LVM index had positive 
Fig. 3. Changes in LVM index and SBP2. The LVM index was significantly higher at the end of the 5-year observation period than at baseline. However, there was no significant change in SBP2 $(\mathrm{n}=$ 52). * Indicates $\mathrm{p}<0.05$.

Suzuki et al.: Decline of Renal Function and Progression of Left Ventricular

Hypertrophy Are Independently Determined in Chronic Kidney Disease Stages 3-5

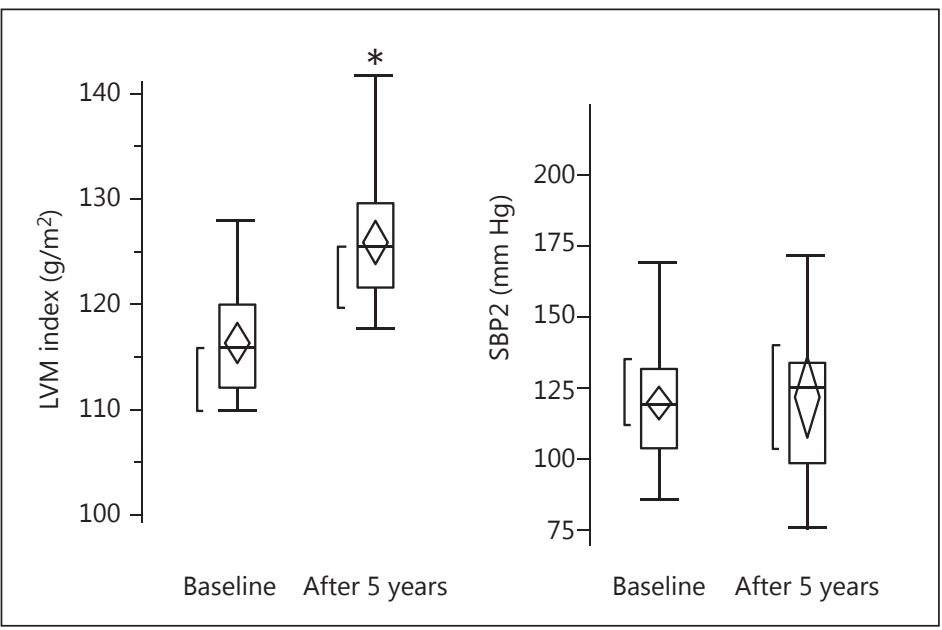

Fig. 4. Kaplan-Meier patients' survival curves. Cumulative proportional patient survival rates at 3 and 5 years were 92 and $89 \%$, respectively.

Table 3. Correlation of various covariates with the decline of renal function

\begin{tabular}{llll}
\hline Variables & r2 & F & p \\
\hline Age (years) & 0.125 & 9.36 & 0.003 \\
SBP (mm Hg) & 0.003 & 0.20 & 0.65 \\
DBP (mm Hg) & 0.028 & 1.91 & 0.17 \\
PP (mm Hg) & 0.004 & 0.27 & 0.60 \\
SBP2 (mm Hg) & 0.002 & 0.14 & 0.65 \\
baPWV (cm/s) & 0.020 & 1.46 & 0.23 \\
Urinary protein excretion (g/g Cr) & 0.025 & 1.87 & 0.20 \\
eGFR (ml/min/1.73 m ${ }^{2)}$ & 0.113 & 9.00 & 0.03 \\
LVM (g/m & 0.053 & 2.26 & 0.14 \\
\hline
\end{tabular}

$\mathrm{Cr}=$ Creatinine .

correlations with baseline SBP2, SBP and PP, and the decline of renal function was correlated with age and the baseline value of eGFR. After multiple forward linear regression analysis, the baseline SBP2 was independently correlated with the increased LVM index ( $<<0.05)$, and the baseline value of eGFR was independently correlated with the decline of renal function $(\mathrm{p}<$ 0.01). 


\begin{tabular}{|c|c|c|c|}
\hline \multicolumn{4}{|l|}{ Pulse 2014;2:29-37 } \\
\hline DOI: $10.1159 / 000368678$ & \multicolumn{3}{|c|}{$\begin{array}{l}\text { (c) } 2014 \text { S. Karger AG, Basel } \\
\text { www.karger.com/pls }\end{array}$} \\
\hline \multicolumn{4}{|c|}{$\begin{array}{l}\text { Suzuki et al.: Decline of Renal Function and Progression of Left Ventricular } \\
\text { Hypertrophy Are Independently Determined in Chronic Kidney Disease Stages 3-5 }\end{array}$} \\
\hline Variables & $\mathrm{r} 2$ & $\mathrm{~F}$ & $\mathrm{p}$ \\
\hline Age (years) & 0.001 & 0.07 & 0.78 \\
\hline SBP (mm Hg) & 0.079 & 3.63 & 0.08 \\
\hline DBP (mm Hg) & 0.039 & 1.75 & 0.19 \\
\hline $\mathrm{PP}(\mathrm{mm} \mathrm{Hg})$ & 0.030 & 1.74 & 0.19 \\
\hline SBP2 (mm Hg) & 0.134 & 6.91 & 0.01 \\
\hline $\mathrm{baPWV}(\mathrm{cm} / \mathrm{s})$ & 0.001 & 0.07 & 0.78 \\
\hline $\mathrm{eGFR}\left(\mathrm{ml} / \mathrm{min} / 1.73 \mathrm{~m}^{2}\right)$ & 0.061 & 3.30 & 0.07 \\
\hline Urinary protein excretion $(\mathrm{g} / \mathrm{g} \mathrm{Cr})$ & 0.065 & 3.33 & 0.07 \\
\hline
\end{tabular}

Table 4. Correlation of various covariates with the increase in LVM index

Table 5. Comparison of laboratory and chocardiographic parameters obtained from the study population $(\mathrm{n}=52)$
$\mathrm{Cr}=$ Creatinine

\begin{tabular}{lcc}
\hline Variables & At baseline & 5 years \\
\hline Albumin, g/dl & $4.1 \pm 0.5$ & $4.0 \pm 0.6$ \\
Total cholesterol, mg/dl & $185.9 \pm 34.8$ & $209.9 \pm 60.23$ \\
Hemoglobin, g/dl & $12.84 \pm 1.69$ & $12.68 \pm 1.89$ \\
Calcium, mg/dl & $9.43 \pm 0.41$ & $8.93 \pm 0.98$ \\
Phosphate, mg/dl & $3.48 \pm 0.73$ & $3.46 \pm 0.83$ \\
Urinary protein excretion, g/g Cr & $1.23 \pm 0.92$ & $1.45 \pm 1.55$ \\
IVST, mm & $10.6 \pm 1.2$ & $10.8 \pm 1.4$ \\
PWT, mm & $9.85 \pm 1.1$ & $10.6 \pm 1.4$ \\
EF, \% & $69.0 \pm 7.0$ & $66.2 \pm 8.5$ \\
\hline
\end{tabular}

$\mathrm{Cr}=$ Creatinine; IVST $=$ intraventricular septum thickness; $\mathrm{PWT}=$ posterior wall thickness; $\mathrm{EF}=$ ejection fraction.

Table 5 shows the comparison of laboratory findings of the 52 patients at the start and at the end of the 5 -year observation period. Also, table 5 shows the parameters obtained from echocardiography at the start and at the end of the 5-year observation period. No parameters showed a significant difference.

\section{Discussion}

This observational study demonstrated a significant increase in LVM index over a 5-year follow-up period in patients with moderately advanced-stage CKD, despite stable BP and normal LV systolic function. This increase might be predictable by the baseline value of SBP2 as an index of central aortic pressure. We also identified that the baseline value of eGFR was an important predictor for progression to ESRD. This notion is generally accepted in advanced CKD patients. However, the decline of renal function did not correlate with any other variables except for baseline age and eGFR at the start of the observation period. The factors that determine or are associated with the decline of renal function have been controversially discussed. Previously, Chue et al. [17] reported that serum phosphate independently predicted a decline in renal function in early CKD but not PWV. In contrast, Kim et al. [18] reported that PP was an independent risk factor for a rapid decline in kidney function in populations with relatively preserved kidney function. Our present findings are similar to those published by $\mathrm{Ng}$ et al. [10] in that the univariate correlations with PWV or LVM index were not significant. $\mathrm{Ng}$ et al. demonstrated that in CKD patients most of the variability in central PP could be 
Suzuki et al.: Decline of Renal Function and Progression of Left Ventricular

Hypertrophy Are Independently Determined in Chronic Kidney Disease Stages 3-5

explained by peripheral PP. Compared with previous reports, our observational period was relatively longer. In the short-term period, several hemodynamic factors such as SBP, PP, and PWV may play some roles; however, over the long term, since these factors are modulated by increases in dose and/or number of antihypertensive drugs, the effects of such hemodynamic factors would be cancelled. There have been no definite studies examining the effects of antihypertensive agents on CAP except the CAFE study [19], in which CAP was the only hemodynamic variable associated with CVD events in hypertensive patients. In line with that study, CAP might be a strong predictor of progression of LVH in hypertensive CKD patients.

Seifert et al. [20] detected a significant increase in LVM, which was not associated with changes in other biomarkers that have been related to worsening CVD risk such as SBP, DBP, and LV systolic function. They proposed an association between the increase in LVM index and klotho expression, the testing of which is not available in general clinical practice.

There are potential limitations to our study. Although prospective, it was observational and cross-sectional in design and therefore subject to potential residual confounding. Another limitation was the lack of available plasma samples to generate data of other biomarkers of progression of LVM, such as fibroblast growth factor 23 , brain natriuretic peptide and so on. The cause of an increase in LVM index is complex and includes various factors. Moreover, a large proportion of patients were receiving antihypertensive medication as is typical of this population. Antihypertensive therapy leads to a decrease of LVM index [21] and PWV [22]. Besides, we did not measure carotid intima-media thickness in our study as a well-established intermediate phenotype. Further, our study was limited by a small patient number. Finally, this study was performed at a single renal center; thus, generalizability of the findings to other populations, including other sites, is unknown; however, this might have cancelled the variable factors produced by multiple centers.

Overall, during the 5-year observation period, $15 \%$ of the participating patients with CKD stages 3-5 developed ESRD. This figure is not high compared with that in general clinical practice in Japan [23]. Also, only 3 patients suffered from CVD during 5 years, indicating that BP control is most important for CKD patients [24]. Moreover, even under well-controlled BP, CAP should be monitored carefully, because LVH is one of the major risk factors for CVD in CKD patients $[25,26]$.

In conclusion, it is suggested that worsening of renal dysfunction is not solely dependent on hemodynamics. Other factors might be involved in a complex manner.

\section{Acknowledgements}

Mrs. Sachiko Nakazato, a secretary, calculated the data and typed the manuscript.

\section{Disclosure Statement}

The authors declare that they have no conflicts of interest in this study.

\section{References}

1 Lorell BH, Carabello BA: Left ventricular hypertrophy: pathogenesis, detection, and prognosis. Circulation 2000;102:470-479.

-2 Glassock RJ, Pecoits-Filho R, Barberato SH: Left ventricular mass in chronic kidney disease and ESRD. Clin J Am Soc Nephrol 2009;4(suppl 1):S79-S91.

-3 Nitta K, Iimuro S, Imai E, Matsuo S, Makino H, Akizawa T, et al: Risk factors for increased left ventricular hypertrophy in patients with chronic kidney disease. Clin Exp Nephrol 2013;17:730-742.

4 Nicholos W, O’Rourke H (eds): Atherosclerosis. London, Annalisa Page, 1998. 
Suzuki et al.: Decline of Renal Function and Progression of Left Ventricular

Hypertrophy Are Independently Determined in Chronic Kidney Disease Stages 3-5

5 Paoletti E, Bellino D, Cassottana P, Rolla D, Cannella G: Left ventricular hypertrophy in nondiabetic predialysis CKD. Am J Kidney Dis 2005;46:320-327.

6 Verdecchia P, Schillaci G, Borgioni C, Gattobigio R, Ambrosio G, Porcellati C: Prevalent influence of systolic over pulse pressure on left ventricular mass in essential hypertension. Eur Heart J 2002;23:658-665.

7 Townsend R, Tomiyama H: Arterial stiffness, kidney function, and chronic kidney disease progression. Pulse 2013;1:123-130.

-8 Taal MW, Sigrist MK, Fakis A, Fluck RJ, McIntyre CW: Markers of arterial stiffness are risk factors for progression to end-stage renal disease among patients with chronic kidney disease stages 4 and 5. Nephron Clin Pract 2007;107:c177-c181.

-9 Upadhyay A, Hwang S-J, Mitchell GF, Vasan RS, Vita JA, Stantchev PI, et al: Arterial stiffness in mild-to-moderate CKD. J Am Soc Nephrol 2009;20:2044-2053.

$\checkmark 10 \mathrm{Ng}$ KP, Moody WE, Chue CD, Edwards NC, Savage T, Tomson CR, et al: Central pulse pressure in patients with chronic kidney disease and in renal transplant recipients. J Hum Hypertens 2014;28:180-185.

-11 KDIGO 2012 Clinical Practice Guideline for the Evaluation and Management of Chronic Kidney Disease. Kidney Int 2013;3(suppl 1):1-119.

12 National Kidney Foundation: K/DOQI clinical practice guidelines for chronic kidney disease: evaluation, classification, and stratification. Am J Kidney Dis 2002;39(2 suppl 1):S1-S266.

13 Matsuo S, Imai E, Horio M, Yasuda Y, Tomita K, Nitta K, et al: Revised equations for estimated GFR from serum creatinine in Japan. Am J Kidney Dis 2009;53:982-992.

14 Suzuki H, Moriwaki K, Nakamoto H, Sugahara S, Kanno Y, Okada H: Blood pressure reduction in the morning yields beneficial effects on progression of chronic renal insufficiency with regression of left ventricular hypertrophy. Clin Exp Hypertens 2002;24:51-63.

15 Nakamura T, Kanno Y, Takenaka T, Suzuki H: An angiotensin receptor blocker reduces the risk of congestive heart failure in elderly hypertensive patients with renal insufficiency. Hypertens Res 2005;28:415-423.

16 Suzuki H, Kobayashi K, Okada H: Combination of echocardiography and pulse wave velocity provides clues for the differentiation between white coat hypertension and hypertension in postmenopausal women. Pulse 2013;1:131-138.

17 Chue CD, Edwards NC, Davis LJ, Steeds RP, Townend JN, Ferro CJ: Serum phosphate but not pulse wave velocity predicts decline in renal function in patients with early chronic kidney disease. Nephrol Dial Transplant 2011; 26:2576-2582.

18 Kim CS, Kim HY, Kang YU, Choi JS, Bae EH, Ma SK, et al: Association of pulse wave velocity and pulse pressure with decline in kidney function. J Clin Hypertens (Greenwich) 2014;16:372-377.

19 Williams B, Lacy PS, Thom SM, Cruickshank K, Stanton A, Collier D, et al: Differential impact of blood pressurelowering drugs on central aortic pressure and clinical outcomes: principal results of the Conduit Artery Function Evaluation (CAFE) study. Circulation 2006;113:1213-1225.

20 Seifert ME, de las Fuentes L, Rothstein M, Dietzen DJ, Bierhals AJ, Cheng SC, et al: Effects of phosphate binder therapy on vascular stiffness in early-stage chronic kidney disease. Am J Nephrol 2013;38:158-167.

21 Boutouyrie P, Achouba A, Trunet P, Laurent S: Amlodipine-valsartan combination decreases central systolic blood pressure more effectively than the amlodipine-atenolol combination: the EXPLOR study. Hypertension 2010;55:1314-1322.

22 DeLoach SS, Appel LJ, Chen J, Joffe MM, Gadegbeku CA, Mohler ER 3rd, et al: Aortic pulse pressure is associated with carotid IMT in chronic kidney disease: report from Chronic Renal Insufficiency Cohort. Am J Hypertens 2009;22:1235-1241.

23 Imai E, Matsuo S, Makino H, Watanabe T, Akizawa T, Nitta K, et al: Chronic Kidney Disease Japan Cohort study: baseline characteristics and factors associated with causative diseases and renal function. Clin Exp Nephrol 2010;14:558-570.

24 Agarwal R: Antihypertensive agents and arterial stiffness: relevance to reducing cardiovascular risk in the chronic kidney disease patient. Curr Opin Nephrol Hypertens 2007;16:409-415.

25 Tsioufis C, Vezali E, Tsiachris D, Dimitriadis K, Taxiarchou E, Chatzis D, et al: Left ventricular hypertrophy versus chronic kidney disease as predictors of cardiovascular events in hypertension: a Greek 6-year-followup study. J Hypertens 2009;27:744-752.

26 Matsumoto M, Io H, Furukawa M, Okumura K, Masuda A, Seto T, et al: Risk factors associated with increased left ventricular mass index in chronic kidney disease patients evaluated using echocardiography. J Nephrol 2012;25:794-801. 